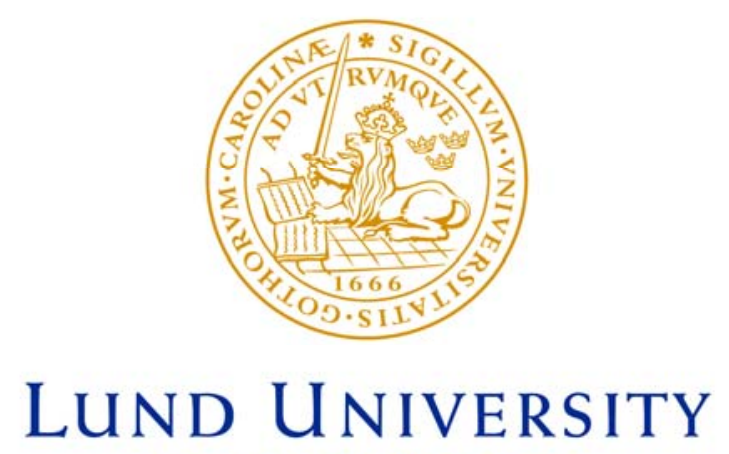

Faculty of Medicine

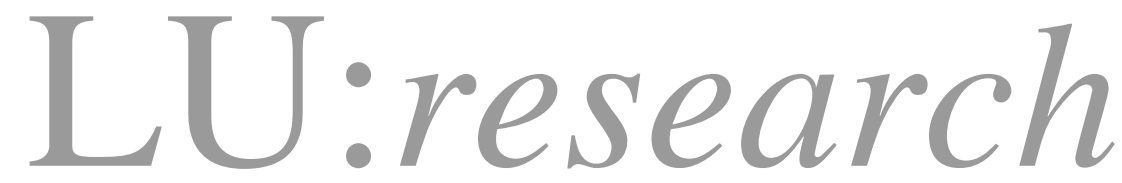

Institutional Repository of Lund University

This is an author produced version of a paper published in Neurobiology of aging. This paper has been peer-reviewed but does not include the final publisher proof-corrections or journal pagination.

Citation for the published paper:

Hansson, Oskar and Buchhave, Peder and Zetterberg, Henrik and Blennow, Kaj and Minthon, Lennart and Warkentin, Siegbert.

"Combined rCBF and CSF biomarkers predict progression from mild cognitive impairment to Alzheimer's disease." Neurobiol Aging, 2007, Issue: Jul 21 [Epub ahead of print]

http://dx.doi.org/10.1016/j.neurobiolaging.2007.06.009

Access to the published version may require journal subscription.

Published with permission from: Elsevier 


\section{Combined rCBF and CSF biomarkers predict progression from mild cognitive impairment to Alzheimer's disease}

Oskar Hansson, MD, PhD, ${ }^{1,2^{*}}$ Peder Buchhave, MD, ${ }^{1,2}$ Henrik Zetterberg, MD, PhD, ${ }^{3,4}$, Kaj Blennow, MD, PhD, ${ }^{3,4}$ Lennart Minthon, MD, PhD, ${ }^{1,2}$ Siegbert Warkentin $\mathrm{PhD}^{1,2}$

${ }^{1}$ Clinical Memory Research Unit, Department of Clinical Sciences Malmö, Lund University, Sweden;

${ }^{2}$ Neuropsychiatric Clinic, Malmö University Hospital, Sweden;

${ }^{3}$ Institute of Clinical Neuroscience, Department of Experimental Neuroscience, Sahlgrenska University Hospital, Göteborg University, Sweden;

${ }^{4}$ Department of Clinical Chemistry and Transfusion Medicine, Sahlgrenska University Hospital, Göteborg University, Sweden

*Correspondence to:

Oskar Hansson, MD, PhD

Neuropsychiatric Clinic

Malmö University Hospital,

S-20502 Malmö, Sweden

Tel: +4640335036

Fax: +46 40334604

E-mail: oskar.hansson@med.lu.se 


\begin{abstract}
This study aimed to identify preclinical Alzheimer's disease (AD) in patients with mild cognitive impairment (MCI) using measurements of both regional cerebral blood flow (rCBF) and cerebrospinal fluid (CSF) biomarkers. Baseline rCBF assessments ( ${ }^{133} \mathrm{Xe}$ method) were performed in 70 patients with MCI who were cognitively stable for 4-6 years, 69 patients with MCI who subsequently developed $\mathrm{AD}$, and 33 healthy individuals. CSF was collected at baseline and analyzed for $\beta$-amyloid - $_{1-42}$, total tau and phophorylated tau. In contrast to patients with stable MCI, those who subsequently developed $\mathrm{AD}$ had decreased $\mathrm{rCBF}$ in the temporo-parietal cortex already at baseline. The relative risk of future progression to AD was particularly increased in MCI patients with decreased $\mathrm{rCBF}$ in parietal cortex (hazard ratio 3.1, $\mathrm{P}<0.0001)$. Patients with pathological levels of both CSF tau and $\beta$-amyloid ${ }_{1-42}$ were also at high risk of developing $\mathrm{AD}$ (hazard ratio 13.4, $\mathrm{P}<0.0001$ ). The combination of decreased parietal rCBF and pathological CSF biomarkers at baseline resulted in a substantially increased risk of future development of $\mathrm{AD}$, with a hazard ratio of $24.3(\mathrm{P}<0.0001)$. Moreover, decreased parietal rCBF (but not CSF biomarkers) was associated with a more rapid progression to $\mathrm{AD}$. In conclusion, the combination of $\mathrm{rCBF}$ and $\mathrm{CSF}$ biomarkers improves the risk assessment of progression to $\mathrm{AD}$ in patients with $\mathrm{MCI}$.
\end{abstract}

Keywords: Mild cognitive impairment; Alzheimer's disease; biomarkers; regional cerebral blood flow; cerebrospinal fluid; tau; beta-amyloid. 


\section{Introduction}

Alzheimer's disease (AD) is the most common cause of dementia [3]. The microscopic hallmarks of $\mathrm{AD}$ are senile plaques, in which $\beta$-amyloid is the major component, together with neurofibrillary tangles containing tau protein. The underlying disease process probably starts decades before the clinical onset of the disease [3]. The first symptoms of subjects with incipient $\mathrm{AD}$ are usually mild impairment of episodic memory. These individuals may fulfill the criteria of mild cognitive impairment (MCI), but they are not yet demented, because they have normal global cognitive functioning and activities of daily living are unaffected [14,30]. However, MCI is a prevalent syndrome in elderly, with a multitude of causes [14,30]. Although approximately $40-60 \%$ of the subjects with MCI develop AD within 4-6 years, many individuals with MCI are cognitively stable over time or even return to a normal functional level [14,30].

Methods to accurately detect incipient AD among subjects with MCI are urgently needed $[3,9]$. Several disease-modifying drugs, which aim to arrest the disease process, are under development, such as $\beta$-amyloid immunotherapy and $\beta$-sheet breakers [3]. Animal studies have shown that such disease-arresting therapies will probably be most efficient in the early stages of the disease, before the neurodegeneration has become too widespread and the subjects are already demented.

The cerebrospinal fluid (CSF) biomarkers tau and $\beta$-amyloid ${ }_{1-42}(\mathrm{~A} \beta 42)$ can be used to detect preclinical AD in MCI populations with relatively high diagnostic accuracy $[4,15,17]$. Measurements of regional cerebral blood flow $(\mathrm{rCBF})$ can also be used to predict progression to $\mathrm{AD}$ in patients with MCI. Several studies using single photon emission computed tomography (SPECT) have shown that decreased $\mathrm{rCBF}$ in temporo-parietal cortex and 
posterior cingulate cortex can identify incipient AD among subjects with $\mathrm{MCI}[6,18-20,22]$. For example, the hitherto largest rCBF study on patients with MCI, comprising 23 patients who developed $\mathrm{AD}$ and 54 patients who were cognitively stable during a follow-up period of 2.2 years, showed that reduced $\mathrm{rCBF}$ in the left parietal cortex can predict $\mathrm{AD}$ with an area under the receiver operating characteristic (ROC) curve of 75\% [20]. Similarly, results from flourodeoxyglucose (FDG)-positron emission tomography (PET) studies have shown decreased metabolism in temporo-parietal regions or posterior cingulate cortex in subjects with MCI who will subsequently develop $\mathrm{AD}[1,8,11,25]$.

The diagnostic accuracy of SPECT for prediction of AD among subjects with MCI tends to be somewhat lower compared with CSF biomarkers [9]. Cerebrospinal fluid tau and A $\beta 42$ are markers of the underlying molecular disease process and they seem to be altered very early in the neuropathological process $[4,17]$. However, these CSF biomarkers do not seem to predict the rate of disease progression, i.e. they are equally changed in MCI subjects who will develop AD within one year as in patients who will convert to AD after e.g. three years [17]. On the other hand, changes in $\mathrm{rCBF}$ become more pronounced as the disease progresses $[19,23]$. Therefore, early changes in rCBF may provide the clinician with important information to identify those MCI patients who will run the risk to develop AD within a relatively short time period.

Only one study has so far assessed the diagnostic value of combining rCBF and CSF biomarkers for the prediction of AD. Okamura and colleagues measured rCBF and CSF tau (but not CSF A 342 ) at baseline in 7 cognitively stable MCI patients and in 17 patients with MCI who developed AD after a mean follow-up of 3 years [27]. The authors showed that a baseline index of CSF tau and $\mathrm{rCBF}$ in posterior cingulate cortex was useful in predicting $\mathrm{AD}$ 
[27]. In the present study, baseline rCBF assessments were performed in $167 \mathrm{MCI}$ subjects, of whom 69 patients developed AD during a follow-up period of 4-6 years (see result section). Thirty three age-matched, healthy elderly were used as controls. Moreover, baseline CSF was analyzed for $\mathrm{A} \beta 42$, total tau and phophorylated tau (P-tau) in $75-76 \%$ of the MCI subjects and $85 \%$ of the controls (see result section). The aims of the present study were the following: 1 ) to confirm the usefulness of rCBF-measurements to predict development of AD by using a larger number of patients with MCI and a longer follow-up time than earlier published studies; 2) to evaluate the added value of combining rCBF with CSF biomarkers to detect incipient $\mathrm{AD}$; and 3) to investigate whether $\mathrm{rCBF}$ or CSF markers can predict the rate of disease progression in the MCI patients who will later on develop AD. 


\section{Materials and methods}

\subsection{MCI patients and healthy controls}

One hundred and sixty seven patients with MCI, who underwent rCBF measurements at baseline, were recruited at Malmö University Hospital, Sweden. At baseline, they underwent physical, neurological and psychiatric examination, careful clinical history and functional assessment. Moreover, computed tomography (CT) of the brain and cognitive tests were performed. The criteria of MCI were those defined by Petersen and collaborators [30,31], which include: (i) memory complaint, preferably corroborated by an informant, (ii) objective memory impairment adjusted for age and education, (iii) preservation of general cognitive functioning (iv) no or minimal impairment of daily life activities, and (v) not fulfilling the DSM-IIIR (Diagnostic and Statistical Manual of Mental Disorders, $3^{\text {rd }}$ edition, revised) criteria of dementia [2]. Patients with other causes of cognitive impairment, including brain tumour, subdural haematoma, CNS infection, and current alcohol abuse were excluded.

The MCI patients were followed clinically at least until they developed a certain type of dementia or until they had been cognitively stable for more than 4 years (average 5.2 years; range 4.0-6.8 years). The patients who received a diagnosis of AD during follow-up were required to meet the DSM-IIIR criteria of dementia [2] and the criteria of probable AD defined by NINCDS-ADRDA (National Institute of Neurological and Communicative Disorders - Stroke/Alzheimer's Disease and Related Disorders Association) [24].

The control population consisted of healthy elderly volunteers, who were recruited in the city of Malmö, Sweden. Inclusion criteria were (i) absence of memory complaints or any other cognitive symptoms, (ii) preservation of general cognitive functioning, and (iii) no active 
neurological or psychiatric disease. The controls were followed clinically for 3 years in order to rule out development of cognitive dysfunction.

Patients gave their informed consent to participate in the study, which was conducted according to the provisions of the Helsinki Declaration and approved by the ethics committee of Lund University, Sweden.

\subsection{Baseline rCBF imaging}

The rCBF was measured by the non-invasive ${ }^{133} \mathrm{Xe}$-inhalation method as described by Obrist and colleagues [26] and Risberg and collaborators [33,34]. This two-dimensional method measures blood flow in cortical areas, while blood flow in deeper structures are not assessed. We used a commersial system with 64 scintillation detectors [NaI (Tl) crystals, $(3 / 4$ ! 3/4)] arranged in a helmet around the head (Cortexplorer 64, Ceretronix, Randers, Denmark). The system adjusts for differences in head size and shapes, and the head positioning is standardized in relation to bony landmarks (nasion and ear channels) by means of light crosses. This makes reposition possible in case of head movements. The measurement procedure was as follows: After a 30-second measurement of the natural background radiation, a mixture of the inert $\gamma$-emitting tracer ${ }^{133} \mathrm{Xe}(90 \mathrm{MBq} / \mathrm{l})$ and air was inhaled by the subject through a facemask for $1 \mathrm{~min}$. During the following period of $10 \mathrm{~min}$, the subject breathed normal air, according to the standard procedure [26]. Thus, the total duration of one rCBF measurement was $11.5 \mathrm{~min}$. The data represent the average blood flow obtained during the washout period. The tracer diffuses into brain tissue from arterial blood and is cleared by venous blood. Blood flow values were calculated from the rate of clearance of the isotope considering also the changes of the arterial concentrations of ${ }^{133} \mathrm{Xe}$, the latter estimated from the isotope concentration in the end-tidal air, sampled from the facemask. The lowest acceptable peak count rates were 1,500 counts/second. The initial slope index (ISI, 2-3 min 
slope measurement) was used, because this flow parameter has shown to have a high stability and reliability also in pathological conditions [33,34]. The arterial partial pressure for $\mathrm{CO}_{2}$ was estimated from the end-tidal $\mathrm{CO}_{2}$ concentrations (Ohmeda gas analyzer), $\mathrm{PE}^{\prime} \mathrm{CO}_{2}$. Measurements of respiration, heart rate and oxygen saturation were performed continuously during the rCBF recordings. Flow values for each detector were calculated as distribution normalized values, based on the hemispheric mean values. A color graphics system (Cortexplorer 64) was used for the presentation of the flow maps. The algorithm for calculation of a given pixel in the maps was based on a weighted linear interpolation of the distance to the four closest detectors. In this way interpolated pixel-by-pixel color-coded maps were created, which show the distribution normalized flow values. Student's t test was used in the analysis of $\mathrm{rCBF}$ decreases in all group comparisons. The statistical parametric rCBFmaps were used to calculate the area of all pixels which showed a significance level of at least $\mathrm{p}<0.05$. The reason for this statistical approach was to retain the possibility to assess small rCBF-differences between the groups, which lie at or above the limit of the methodological error (the test-retest stability of the present Xe-method is about $1 \%$, unpublished data). The total area of these significant pixels (i.e. flow decreases) was calculated as per cent of the respective hemisphere in which they were located. The statistically significant flow difference was calculated for each individual MCI-patient compared with the normal elderly subject group. The complete area analyses included separate assessments of temporal lobes, parietal lobes, as well as in the total cortex (i.e. right plus left hemsipheres). The color-coded illustrations in Fig. 1 show the location and the extent of the significant flow decreases in several group comparisons.

\subsection{Analysis of baseline CSF}


CSF was collected in polypropylene tubes, stored at $-80{ }^{\circ} \mathrm{C}$ and analysed after the clinical follow-up of the study was completed. The levels of total tau, tau phosphorylated at $\mathrm{Thr}_{181}(\mathrm{P}-$ tau) and $\beta$-amyloid 1-42 $_{\text {(A } \beta 42)}$ were determined using xMAP technology as previously described $[17,28]$. Briefly, this technology is based on flow cytometric separation of antibodycoated microspheres, which are labelled with a mixture of two fluorescent dyes. After binding of a reporter antibody, quantification is made by adding of a third fluorochrome. The technique permits measurement of several analytes at the same time in the same reaction and the results correlate very well with conventional ELISA measurements [28].

\subsection{Statistical analyses}

To compare demographic and CSF baseline data between groups, a non-parametric KruskalWallis ANOVA was performed followed by Mann-Whitney $U$-test for continuous variables. Pearson's $x^{2}$ test was used for dichotomous variables. The Spearman correlation coefficient was used for bivariate correlation analyses. In the correlation analyses of the associations between $\mathrm{rCBF}$ and CSF biomarkers, the areas with significantly decreased $\mathrm{rCBF}$ in the parietal cortex were not adjusted for potential brain volume loss. Cox proportional hazards models were used to independently estimate the effects of different baseline risk factors on the relative hazard of progression from MCI to AD. The analyses were carried out with and without adjustment for potential confounding of the baseline demographic variables, i.e. age, gender, MMSE and $A P O E \& 4$ carrier status. The statistical analyses were accomplished with SPSS for Windows, version 12.0.1. Receiver operating characteristic (ROC) curves were drawn by plotting the true-positive fraction (sensitivity) against false-positive fraction (100\% - specificity). The area under the curve (AUC) and the confidence interval of $95 \%$ were calculated using MedCalc for Windows, version 8.2 (Medcalc Software, Mariakerke, Belgium). The MedCalc software was also used to assess the statistical difference of 
diagnostic performance between the decreased $\mathrm{rCBF}$ in different temporo-parietal regions according to the method developed by Hanley and McNeil [16]. 


\section{Results}

\subsection{Subjects}

Out of the $167 \mathrm{MCI}$ subjects with rCBF measurements obtained at baseline, 70 patients $(42 \%)$ were cognitively stable when followed for more than 4 years (mean follow-up time, 5.2 years; range, 4.0-6.8 years). Three subjects (2\%) died before 4 years of follow-up. Due to uncertainty of their cognitive stability they were excluded from the study. During the 4-6 years of follow-up, 69 of the subjects with MCI at baseline (41\%) developed AD. Moreover, 25 MCI patients (15\%) progressed to other forms of dementia that included vascular dementia (18 subjects), dementia with Lewy bodies (4 subjects), semantic dementia (1 subject), frontotemporal dementia (1 subject) and traumatic brain injury-induced dementia (1 subject). As the group of subjects who developed other forms of dementia than $\mathrm{AD}$ was relatively small and heterogeneous, the data from these patients were not analyzed further in this study. Moreover, 33 age-matched healthy individuals, of whom none developed dementia within 3 years of follow-up, were used as controls. Demographic data of the included subjects are presented in table 1. Statistical analyses confirmed established risk factors for development of $\mathrm{AD}$ among MCI subjects, namely high age, female gender and positive $A P O E \& 4$ carrier status (tables 1 and 3 ).

\subsection{Baseline $r C B F$ for the prediction of $A D$}

Patients with MCI, who subsequently developed AD, showed significantly lower rCBF-values in parietal and superior temporal cortical areas at baseline, when compared both to stable MCI patients and healthy elderly controls (figure 1, table 2). Receiver operating characteristic (ROC) analysis was performed to determine the power of decreased rCBF in different temporo-parietal regions to discriminate between the MCI patients who developed AD during follow-up from those MCI patients who were cognitively stable (figure $2 \mathrm{~A}$ ). The $\mathrm{rCBF}$ in the 
left parietal cortex showed the best diagnostic accuracy with $77.2 \%$ of the area under the ROC curve $(P<0.0001)$ (figure $2 \mathrm{~A})$. When comparing the diagnostic accuracy of rCBF-decreases in the separate temporal and parietal regions, the area under the ROC curve was significantly larger for the left parietal $\mathrm{rCBF}$ compared with the $\mathrm{rCBF}$ in the right temporal region $(P<$ $0.05)$ and the left temporal region $(P<0.05)$ (figure $2 \mathrm{~A})$. Further, when analyzing rCBF in both right and left hemispheres simultaneously, we found that the bilateral parietal rCBF decrease could predict AD with an area under the ROC curve of $78.1 \%(P<0.0001)$ (figure 2B). The area under the ROC curve was significantly larger for the bilateral parietal rCBF compared with the bilateral temporal $\mathrm{rCBF}(P<0.05)$ (figure $2 \mathrm{~B})$.

Figure 3A illustrates the Kaplan-Meier estimates of the probability of conversion to AD in MCI patients with either normal or pathological $\mathrm{rCBF}$ at baseline, where pathological $\mathrm{rCBF}$ was defined as a significantly decreased cortical $\mathrm{rCBF}$ in the parietal areas representing more than $1.5 \%$ of the total cerebral cortex. The univariate Cox proportional hazards model revealed that there was a significant difference in the probability of progression from MCI to $\mathrm{AD}$ between the subjects with pathological $\mathrm{rCBF}$ and those with normal $\mathrm{rCBF}$ at baseline (hazard ratio $3.92,95 \% \mathrm{CI} 2.41$ to $6.38, P<0.0001$ ) (table 3; figure 3A). Decreased parietal rCBF remained a significant risk factor for future development of $\mathrm{AD}$ even after adjustment for possible demographic risk factors for AD such as age, gender, MMSE and $A P O E$ genotype (adjusted hazard ratio $3.14 ; 95 \%$ CI, 1.90 to 5.20; $P<0.001$ ) (table 3).

\subsection{Combined baseline $\mathrm{rCBF}$ and $C S F$ analyses for the prediction of $A D$}

Out of the 70 cognitively stable MCI subjects who underwent rCBF measurements, CSF was obtained at baseline in 53 subjects (76\%), and out of the 69 MCI subjects who developed AD, CSF was obtained at baseline in 52 subjects (75\%) (table 2). Moreover, lumbar puncture was 
also performed in 28 age-matched healthy elderly controls (85\%) (table 2). Patients who did not undergo lumbar puncture did not differ from those who underwent the procedure with respect to diagnosis after clinical follow-up, age, sex, baseline MMSE score, annual change of MMSE during follow-up, or any rCBF measurements. The relative risk of progression to $\mathrm{AD}$ was increased in patients with MCI who had pathological concentrations of both CSF A $\beta 42$ $(<530 \mathrm{ng} / \mathrm{l})$ and CSF total tau (>350 ng/l) at baseline (adjusted hazard ratio 13.4; 95\% CI, 4.00 to $45.1 ; P<0.001$ ) (table 3). In addition, figure 3B shows the Kaplan-Meier estimates of the probability of conversion to AD in MCI subjects with either 1. normal CSF, 2.

pathological CSF and normal rCBF or, 3. pathological CSF and pathological rCBF. The MCI patients with a combination of decreased parietal $\mathrm{rCBF}$ and pathological CSF biomarkers at baseline had a substantially increased risk of future AD, with an adjusted hazard ratio of 24.3 (95\% CI, 6.98-84.6, $P<0.0001$ ), when compared to those with normal CSF (table 3) (figure 3B). Inclusion of P-tau in the definition of pathological CSF (T-tau $>350 \mathrm{ng} / \mathrm{L}$ and Abeta42/P-tau $<6.5$ ) did not change these results significantly (data not shown)

\subsection{Correlation between disease progression and baseline $r C B F$}

Among the patients with MCI, who subsequently developed AD, there were significant correlations between the parietal area with significantly decreased $\mathrm{rCBF}$ at baseline and 1) the time to conversion to $\mathrm{AD}(r=-0.31 ; P<0.01)$ and 2$)$ the annual decrease of MMSE score during follow-up $(r=0.31 ; P<0.01)$. That is, MCI patients with a larger area of significantly decreased $\mathrm{rCBF}$ in the parietal cortex at baseline also had a more rapid progression to AD dementia. In contrast, in the same patient group there were no significant relations between the levels of CSF biomarkers (total tau, P-tau or A $\beta 42$ ) and the time period to conversion to $\mathrm{AD}$ or the annual decrease of MMSE during follow-up $(\mathrm{P}>0.05)$. Further, when analyzing controls, MCI patients who later developed $\mathrm{AD}$ and cognitively stable MCI patients 
simultaneously, there were significant correlations between the parietal area with significantly decreased rCBF and 1) CSF tau $(r=0.30 ; P<0.001), 2)$ CSF P-tau $(r=0.27 ; P<0.001)$ and 3) CSF A $442(r=-0.38 ; P<0.001)$. That is, subjects with a larger area of significantly decreased rCBF in the parietal cortex at baseline also had higher levels of CSF tau and CSF Ptau, but lower levels of CSF A $\beta 42$. However, when the different subgroups were evaluated separately, no associations were found between CSF biomarkers and parietal rCBF. 


\section{Discussion}

We conducted a relatively large clinically based study, evaluating the use of combining rCBF and CSF biomarkers in patients with MCI to assess the risk of future development of AD. The relative risk of progression to $\mathrm{AD}$ was increased in patients with $\mathrm{MCI}$ who had decreased rCBF in parietal cortex at baseline (adjusted hazard ratio 3.1). Furthermore, the MCI patients with pathological levels of both CSF A $\beta 42$ and tau at baseline were at high risk of developing $\mathrm{AD}$ (adjusted hazard ratio 13.4). The combination of decreased parietal $\mathrm{rCBF}$ and pathological CSF biomarkers resulted in a further increased risk of future AD (adjusted hazard ratio 24.3). Moreover, the degree of parietal rCBF decrease, but not CSF biomarkers, was associated with a more rapid development of Alzheimer's dementia.

Biomarkers for neurodegenerative disorders can be divided into markers of disease state and markers of disease rate [13]. Markers of disease state, usually called diagnostic biomarkers, should reflect the underlying pathogenic process and facilitate detection of a certain biological disease in populations of individuals with similar symptoms (e.g. memory impairment) caused by different conditions. Such markers should also be able to predict the risk of future clinical symptoms. However, markers of the rate of disease progression, which can be used to track changes over time, will be very useful when studying the efficacy of new disease-modifying therapies [10]. It is quite unlikely that a single marker will fulfil the roles of measuring both the state and rate of $\mathrm{AD}[13,35]$. In the absence of a single ideal diagnostic test, combining biomarkers might prove to increase diagnostic precision $[13,35]$. We and others have previously shown that the CSF biomarkers tau and A $\beta 42$ can be used to detect preclinical AD in populations with MCI $[4,15,17]$. Several other methods with the potential to directly image key aspects of the pathophysiology of $\mathrm{AD}$ could be used to predict future development of $\mathrm{AD}$, including amyloid imaging, functional MRI, FDG-PET and rCBF assessments [3,9,10,13]. 
Despite these possibilities, only little is known about the added diagnostic value of combining different biomarkers [27]. This article provides proof of principle that a combination of rCBF and CSF biomarkers clearly improves the risk assessment of future AD in subjects with MCI. Moreover, the results of the present study imply that $\mathrm{rCBF}$, but not CSF biomarkers, can be used to predict an earlier onset of AD dementia. These findings are in agreement with studies showing that $\mathrm{rCBF}$ decreases progress substantially with the AD disease process $[19,23,36]$, in contrast to CSF biomarkers, which are quite stable over time in individual AD patients [5]. Therefore, $\mathrm{rCBF}$ measurements provide additional information in the clinical setting regarding prediction of the disease progression rate of $\mathrm{AD}$.

The results of the present study have shown that decreased rCBF in the parietal cortex provides a high diagnostic accuracy. This is in accordance with the majority of previous studies, showing that functional abnormalities in the parietal cortex have the highest predictive values for the future development of $\mathrm{AD}$ compared to other brain regions $[1,6,8,18$ 20,25]. However, this is not an unequivocal finding as some other studies have suggested that decreased $\mathrm{rCBF}$ in the posterior cingulated gyrus is superior in detecting incipient $\mathrm{AD}$ among subjects with MCI $[22,23,27]$.The reason for this discrepancy is unknown, although differences in instrumentation and variations in the affection of brain areas in this heterogeneous population of subjects with MCI may be possible explanations. From a methodological point of view, the predictive accuracy of cortical rCBF obtained with the present ${ }^{133}$ Xe method was very similar to previously reported SPECT studies [6,18-20]. In fact, the reported areas under the ROC curves for the power of SPECT to predict AD in MCI populations show a range between 74 to $82 \%[7,12,19-21]$, which is quite similar to the $78 \%$ we found for parietal $\mathrm{rCBF}$ in the present study. As methodological differences between studies may contribute to the variation in predictive accuracies, further studies are needed to 
evaluate if a combination of CSF biomarkers with other brain imaging methods will result in even better diagnostic risk assessment.

To our knowledge, this study represents the largest sample of subjects with MCI and the most extensive clinical follow-up time presented this far. Long follow-up time of cognitively stable MCI subjects and controls is very important in order to reduce the number of individuals in these groups who will develop $\mathrm{AD}$ in the future. Therefore, even longer follow-up time of the MCI patients of the present study might have improved the results further. Moreover, the current study is the first to combine the CSF biomarkers $\mathrm{A} \beta 42$ and tau with rCBF. However, a limitation of the study might be that some of the patients that underwent $\mathrm{rCBF}$ at baseline did not undergo lumbar puncture (15-25\%). Nevertheless, this should not significantly affect the results of the study, because the patients who did not undergo lumbar puncture did not differ from those who underwent the procedure with respect to diagnosis after clinical follow-up or any important demographic data.

The results of the present study may have an impact on the diagnostic work-up of subjects with MCI, if they are corroborated by other large studies with long follow-up time that combine CSF and rCBF biomarkers. In the future, individuals with mild memory impairment and a combination of pathological $\mathrm{rCBF}$ and CSF markers need to be followed carefully in the clinic. Moreover, these results may have an influence on the design of clinical trials aiming at investigating the potential disease-arresting effects of new $\mathrm{AD}$ therapies in populations of subjects with MCI. In former drug trials as many as $50-80 \%$ of the MCI subjects did not develop AD during a follow-up of 3-4 years [32,37]. In such heterogeneous populations it might be more difficult to detect positive treatment effects. Moreover, it may be regarded unethical to include large numbers of patients who do not have incipient $\mathrm{AD}$ in treatment 
trials, considering the potential side effects of new therapies such as A $\beta 42$ immunotherapy [29].

In conclusion, this study shows that MCI subjects with both decreased parietal $\mathrm{rCBF}$ and pathological levels of CSF tau and A $\beta 42$ are at very high risk of developing AD in the future. Moreover, decreased parietal rCBF may be associated with a more rapid development of the disease. However, further longitudinal studies on MCI patients are warranted to confirm these results.

\section{Acknowledgements}

This work was supported by the Swedish Alzheimer foundation, the Segerfalk Foundation, Stiftelsen Gamla Tjänarinnor, the Swedish Research Council and Skane county council's research and development foundation.

\section{Disclosure Statement}

The authors have no conflicts of interest. 


\section{References}

[1] Arnaiz E, Jelic V, Almkvist O, Wahlund LO, Winblad B, Valind S, Nordberg A. Impaired cerebral glucose metabolism and cognitive functioning predict deterioration in mild cognitive impairment. Neuroreport 2001;12(4):851-5.

[2] Association AP. Diagnostic and Statistical Manual of Mental Disorders, third edn, revised. Arlington, VA, USA: American Psychiatric Association; 1987.

[3] Blennow K, de Leon MJ, Zetterberg H. Alzheimer's disease. Lancet 2006;368(9533):387-403.

[4] Blennow K, Hampel H. CSF markers for incipient Alzheimer's disease. Lancet Neurol 2003;2(10):605-13.

[5] Blennow K, Zetterberg H, Minthon L, Lannfelt L, Strid S, Annas P, Basun H, Andreasen N. Longitudinal stability of CSF biomarkers in Alzheimer's disease. Neurosci Lett 2007;419(1):18-22.

[6] Borroni B, Anchisi D, Paghera B, Vicini B, Kerrouche N, Garibotto V, Terzi A, Vignolo LA, Di Luca M, Giubbini R, Padovani A, Perani D. Combined 99mTc-ECD SPECT and neuropsychological studies in MCI for the assessment of conversion to AD. Neurobiol Aging 2006;27(1):24-31.

[7] Borroni B, Perani D, Broli M, Colciaghi F, Garibotto V, Paghera B, Agosti C, Giubbini R, Di Luca M, Padovani A. Pre-clinical diagnosis of Alzheimer disease combining platelet amyloid precursor protein ratio and $\mathrm{rCBF}$ spect analysis. J Neurol 2005;252(11):1359-62.

[8] Chetelat G, Eustache F, Viader F, De La Sayette V, Pelerin A, Mezenge F, Hannequin D, Dupuy B, Baron JC, Desgranges B. FDG-PET measurement is more accurate than neuropsychological assessments to predict global cognitive deterioration in patients with mild cognitive impairment. Neurocase 2005;11(1):14-25.

[9] Chong MS, Sahadevan S. Preclinical Alzheimer's disease: diagnosis and prediction of progression. Lancet Neurol 2005;4(9):576-9.

[10] Dickerson BC, Sperling RA. Neuroimaging biomarkers for clinical trials of diseasemodifying therapies in Alzheimer's disease. NeuroRx 2005;2(2):348-60.

[11] Drzezga A, Grimmer T, Riemenschneider M, Lautenschlager N, Siebner H, Alexopoulus P, Minoshima S, Schwaiger M, Kurz A. Prediction of individual clinical outcome in MCI by means of genetic assessment and (18)F-FDG PET. J Nucl Med 2005;46(10):1625-32.

[12] Encinas M, De Juan R, Marcos A, Gil P, Barabash A, Fernandez C, De Ugarte C, Cabranes JA. Regional cerebral blood flow assessed with 99mTc-ECD SPET as a marker of progression of mild cognitive impairment to Alzheimer's disease. Eur J Nucl Med Mol Imaging 2003;30(11):1473-80.

[13] Fox N, Growdon JH. Biomarkers and surrogates. NeuroRx 2004;1(181):181.

[14] Gauthier S, Reisberg B, Zaudig M, Petersen RC, Ritchie K, Broich K, Belleville S, Brodaty H, Bennett D, Chertkow H, Cummings JL, de Leon M, Feldman H, Ganguli M, Hampel H, Scheltens P, Tierney MC, Whitehouse P, Winblad B. Mild cognitive impairment. Lancet 2006;367(9518):1262-70.

[15] Hampel H, Teipel SJ, Fuchsberger T, Andreasen N, Wiltfang J, Otto M, Shen Y, Dodel R, Du Y, Farlow M, Moller HJ, Blennow K, Buerger K. Value of CSF betaamyloid1-42 and tau as predictors of Alzheimer's disease in patients with mild cognitive impairment. Mol Psychiatry 2004;9(7):705-10.

[16] Hanley JA, McNeil BJ. A method of comparing the areas under receiver operating characteristic curves derived from the same cases. Radiology 1983;148(3):839-43. 
[17] Hansson O, Zetterberg H, Buchhave P, Londos E, Blennow K, Minthon L. Association between CSF biomarkers and incipient Alzheimer's disease in patients with mild cognitive impairment: a follow-up study. Lancet Neurol 2006;5(3):228-34.

[18] Hirao K, Ohnishi T, Hirata Y, Yamashita F, Mori T, Moriguchi Y, Matsuda H, Nemoto K, Imabayashi E, Yamada M, Iwamoto T, Arima K, Asada T. The prediction of rapid conversion to Alzheimer's disease in mild cognitive impairment using regional cerebral blood flow SPECT. Neuroimage 2005;28(4):1014-21.

[19] Huang C, Eidelberg D, Habeck C, Moeller J, Svensson L, Tarabula T, Julin P. Imaging markers of mild cognitive impairment: Multivariate analysis of CBF SPECT. Neurobiol Aging 2006.

[20] Huang C, Wahlund LO, Almkvist O, Elehu D, Svensson L, Jonsson T, Winblad B, Julin P. Voxel- and VOI-based analysis of SPECT CBF in relation to clinical and psychological heterogeneity of mild cognitive impairment. Neuroimage 2003;19(3):1137-44.

[21] Huang C, Wahlund LO, Svensson L, Winblad B, Julin P. Cingulate cortex hypoperfusion predicts Alzheimer's disease in mild cognitive impairment. BMC Neurol 2002;2:9.

[22] Johnson KA, Jones K, Holman BL, Becker JA, Spiers PA, Satlin A, Albert MS. Preclinical prediction of Alzheimer's disease using SPECT. Neurology 1998;50(6):1563-71.

[23] Kogure D, Matsuda H, Ohnishi T, Asada T, Uno M, Kunihiro T, Nakano S, Takasaki M. Longitudinal evaluation of early Alzheimer's disease using brain perfusion SPECT. J Nucl Med 2000;41(7):1155-62.

[24] McKhann G, Drachman D, Folstein M, Katzman R, Price D, Stadlan EM. Clinical diagnosis of Alzheimer's disease: report of the NINCDS-ADRDA Work Group under the auspices of Department of Health and Human Services Task Force on Alzheimer's Disease. Neurology 1984;34(7):939-44.

[25] Mosconi L, Perani D, Sorbi S, Herholz K, Nacmias B, Holthoff V, Salmon E, Baron JC, De Cristofaro MT, Padovani A, Borroni B, Franceschi M, Bracco L, Pupi A. MCI conversion to dementia and the APOE genotype: a prediction study with FDG-PET. Neurology 2004;63(12):2332-40.

[26] Obrist WD, Thompson HK, Jr., Wang HS, Wilkinson WE. Regional cerebral blood flow estimated by 133-xenon inhalation. Stroke 1975;6(3):245-56.

[27] Okamura N, Arai H, Maruyama M, Higuchi M, Matsui T, Tanji H, Seki T, Hirai H, Chiba H, Itoh M, Sasaki H. Combined Analysis of CSF Tau Levels and [(123)I]Iodoamphetamine SPECT in Mild Cognitive Impairment: Implications for a Novel Predictor of Alzheimer's Disease. Am J Psychiatry 2002;159(3):474-6.

[28] Olsson A, Vanderstichele H, Andreasen N, De Meyer G, Wallin A, Holmberg B, Rosengren L, Vanmechelen E, Blennow K. Simultaneous measurement of betaamyloid(1-42), total tau, and phosphorylated tau (Thr181) in cerebrospinal fluid by the xMAP technology. Clin Chem 2005;51(2):336-45.

[29] Orgogozo JM, Gilman S, Dartigues JF, Laurent B, Puel M, Kirby LC, Jouanny P, Dubois B, Eisner L, Flitman S, Michel BF, Boada M, Frank A, Hock C. Subacute meningoencephalitis in a subset of patients with AD after Abeta42 immunization. Neurology 2003;61(1):46-54.

[30] Petersen RC. Mild cognitive impairment as a diagnostic entity. J Intern Med 2004;256(3):183-94.

[31] Petersen RC, Smith GE, Waring SC, Ivnik RJ, Tangalos EG, Kokmen E. Mild cognitive impairment: clinical characterization and outcome. Arch Neurol 1999;56(3):303-8. 
[32] Petersen RC, Thomas RG, Grundman M, Bennett D, Doody R, Ferris S, Galasko D, Jin S, Kaye J, Levey A, Pfeiffer E, Sano M, van Dyck CH, Thal LJ. Vitamin E and donepezil for the treatment of mild cognitive impairment. N Engl J Med 2005;352(23):2379-88.

[33] Risberg J. Regional cerebral blood flow measurements by 133Xe-inhalation: methodology and applications in neuropsychology and psychiatry. Brain Lang 1980;9(1):9-34.

[34] Risberg J, Gustafson L. Regional cerebral blood flow measurements in the clinical evaluation of demented patients. Dement Geriatr Cogn Disord 1997;8(2):92-7.

[35] Schott JM, Kennedy J, Fox NC. New developments in mild cognitive impairment and Alzheimer's disease. Curr Opin Neurol 2006;19(6):552-8.

[36] Silverman DH, Small GW, Chang CY, Lu CS, Kung De Aburto MA, Chen W, Czernin J, Rapoport SI, Pietrini P, Alexander GE, Schapiro MB, Jagust WJ, Hoffman JM, Welsh-Bohmer KA, Alavi A, Clark CM, Salmon E, de Leon MJ, Mielke R, Cummings JL, Kowell AP, Gambhir SS, Hoh CK, Phelps ME. Positron emission tomography in evaluation of dementia: Regional brain metabolism and long-term outcome. Jama 2001;286(17):2120-7.

[37] Thal LJ, Ferris SH, Kirby L, Block GA, Lines CR, Yuen E, Assaid C, Nessly ML, Norman BA, Baranak CC, Reines SA. A randomized, double-blind, study of rofecoxib in patients with mild cognitive impairment. Neuropsychopharmacology 2005;30(6):1204-15. 


\section{Figure legends}

\section{Figure 1}

Upper row shows the cortical areas with significantly decreased rCBF at baseline in subjects with MCI who subsequently developed AD (MCI-AD, n=69) compared with healthy elderly controls $(n=33)$. The rCBF of parietal and superior temporal areas of both hemispheres were significantly lower in the MCI-AD subjects compared with normal aging. Lower row shows the cortical areas with significantly decreased $\mathrm{rCBF}$ at baseline in MCI-AD subjects $(n=69)$ compared with those subjects with MCI who remained cognitively stable during 4-6 years of follow-up (Stable MCI, $n=70$ ). In this comparison, MCI subjects who later developed AD had significantly lower rCBF in temporal and parietal areas of both hemispheres at baseline.

\section{Figure 2}

Receiver operating characteristic (ROC) curves of the power of reduced $\mathrm{rCBF}$ in different temporo-parietal regions at baseline, to discriminate between the MCI patients who subsequently developed $\mathrm{AD}(\mathrm{n}=69)$ from the MCI patients who were cognitively stable during 4-6 years of follow-up ( $n=70)$. Panel A: The $\mathrm{rCBF}$ in the left parietal cortex showed a satisfactory diagnostic accuracy with $77.2 \%$ of the area under the ROC curve (AUC). The AUCs for the rCBF in the right parietal cortex, the left temporal cortex and the right temporal cortex were $71.0 \%, 66.0 \%$ and $61.3 \%$, respectively. There was a statistically significant difference in the $\mathrm{AUC}$ between the left parietal $\mathrm{rCBF}$ and the $\mathrm{rCBF}$ in 1) the right temporal region $(P<0.05)$ and 2$)$ the left temporal region $(P<0.05)$. Panel B: The $\mathrm{rCBF}$ was analyzed in both right and left hemispheres simultaneously. The area under the ROC curve was significantly larger for the bilateral parietal $\mathrm{rCBF}(\mathrm{AUC}=78.1 \%)$ compared with the bilateral temporal $\mathrm{rCBF}(\mathrm{AUC}=66.8 \%)(P<0.05)$. 


\section{Figure 3}

Panel A depicts Kaplan-Meier estimates of the rate of progression to AD in MCI subjects with either normal rCBF or pathological $\mathrm{rCBF}$ at baseline. Among the $69 \mathrm{MCI}$ subjects who subsequently developed $\mathrm{AD}$ and the 70 cognitively stable MCI subjects, all of whom underwent $\mathrm{rCBF}$ at baseline, 97 had normal $\mathrm{rCBF}$ and 42 had pathological rCBF. Panel B shows Kaplan-Meier estimates of the rate of progression to AD in MCI subjects with either 1) normal CSF; 2) pathological CSF and normal rCBF; or 3) pathological CSF and pathological rCBF at baseline. Among the MCI subjects, who underwent both rCBF and lumbar puncture at baseline, 52 subsequently developed $\mathrm{AD}$ and 53 were cognitively stable. Out of these subjects, 40 had normal CSF and normal rCBF; 6 had normal CSF and pathological rCBF; 33 pathological CSF and normal rCBF; and 26 had pathological CSF and pathological rCBF. Individuals with normal CSF were analyzed as one group $(n=46)$. Pathological rCBF was defined as a significantly decreased cortical $\mathrm{rCBF}$ in the parietal areas representing more than $1.5 \%$ of the total cerebral cortex. The definition of pathological CSF was a combination of CSF total tau $>350 \mathrm{ng} / \mathrm{L}$ and CSF A $\beta 42<530 \mathrm{ng} / \mathrm{L}$. 
Table 1. Baseline demographic data and annual change of MMSE during follow-up of the patients with MCI and healthy controls, who underwent rCBF at baseline.

\begin{tabular}{|c|c|c|c|}
\hline & Controls & Stable MCI & MCI-AD \\
\hline Male/Female & $11 / 22$ & $37 / 33$ & $18 / 51^{b}$ \\
\hline Age, mean (range) & $74(60-94)$ & $65(50-82)^{\mathrm{a}}$ & $75(59-87)^{\mathrm{C}}$ \\
\hline APOE $\varepsilon 4$ carrier, $\%$ & 21 & 42 & $68^{\mathrm{a}, \mathrm{b}}$ \\
\hline MMSE at baseline & $29.1(1.3)$ & $27.4(1.9)^{\mathrm{a}}$ & $26.6(1.4)^{\mathrm{a}, \mathrm{b}}$ \\
\hline $\begin{array}{l}\text { Annual change of MMSE } \\
\text { during follow-up }\end{array}$ & $+0.10(0.41)$ & $+0.19(0.42)$ & $-3.1(2.1)^{\mathrm{a}, \mathrm{c}}$ \\
\hline
\end{tabular}

Values are means (SD), except as noted otherwise.

${ }^{\mathrm{a}} \mathrm{P}<0.001$ vs Controls, ${ }^{\mathrm{b}} \mathrm{P}<0.01$ vs Stable MCI, ${ }^{\mathrm{c}} \mathrm{P}<0.001$ vs Stable MCI

Abbreviations: Stable MCI, MCI patients with stable cognitive functions during a follow-up period of 4.0-6.8 years; MCI-AD, MCI patients who developed Alzheimer's disease during follow-up; APOE, apolipoprotein E; MMSE, Mini-Mental State Examination. 
Table 2. CSF biomarkers and the sizes of cortical areas within the temporal and parietal lobes with significantly decreased $\mathrm{rCBF}$ at baseline.

\begin{tabular}{|l|l|l|l|}
\hline \multicolumn{1}{|c|}{ rCBF } & Controls & Stable MCI & MCI-AD \\
\hline Left parietal lobe* & $\mathrm{n}=33$ & $\mathrm{n}=70$ & $\mathrm{n}=69$ \\
\hline Right parietal lobe* & $0.22(0.61)$ & $0.40(0.88)$ & $2.1(2.2)^{\mathrm{b}, \mathrm{d}}$ \\
\hline Left temporal lobe* & $0.34(0.91)$ & $0.63(1.3)$ & $2.0(2.3)^{\mathrm{b}, \mathrm{d}}$ \\
\hline Right temporal lobe* & $0.39(0.88)$ & $0.81(1.8)$ & $2.5(3.2)^{\mathrm{a}, \mathrm{d}}$ \\
\hline Bilateral parietal lobes** & $0.28(0.66)$ & $0.90(1.7)$ & $1.9(2.8)^{\mathrm{b}, \mathrm{c}}$ \\
\hline Bilateral temporal lobes** & $0.55(1.0)$ & $0.52(0.94)$ & $2.0(1.9)^{\mathrm{b}, \mathrm{d}}$ \\
\hline \multicolumn{1}{|c|}{ CSF } & $\mathrm{n}=28$ & $0.86(1.4)$ & $2.2(2.6)^{\mathrm{b}, \mathrm{d}}$ \\
\hline Total tau (ng/l) & $380(217)$ & $\mathrm{n}=53$ & $\mathrm{n}=52$ \\
\hline P-tau (ng/l) & $61(18)$ & $331(207)$ & $819(429)^{\mathrm{b}, \mathrm{d}}$ \\
\hline A $\beta 42(\mathrm{ng} / \mathrm{l})$ & $738(160)$ & $62(16)$ & $94(29)^{\mathrm{b}, \mathrm{d}}$ \\
\hline
\end{tabular}

Values are means \pm SD.

*The areas are expressed as percentage of total cortical area in the same hemisphere.

** The areas are expressed as percentage of total cortical area of both hemispheres.

${ }^{\mathrm{a}} P<0.05$ vs Controls, ${ }^{\mathrm{b}} P<0.001$ vs Controls, ${ }^{\mathrm{c}} P<0.05$ vs Stable MCI, ${ }^{\mathrm{d}} P<0.001$ vs Stable MCI

Abbreviations: Stable MCI, MCI patients with stable cognitive functions during a follow-up period of 4.0-6.8 years; MCI-AD, MCI patients that developed Alzheimer's disease during follow-up; P-tau, phosphorylated tau; A $\beta 42, \beta$-amyloid $1-42$. 
Table 3. Cox proportional-hazards regression models examining the relation between baseline risk factors, including $\mathrm{rCBF}$ and $\mathrm{CSF}$ biomarkers, and the risk of converting to $\mathrm{AD}$ in patients with MCI.

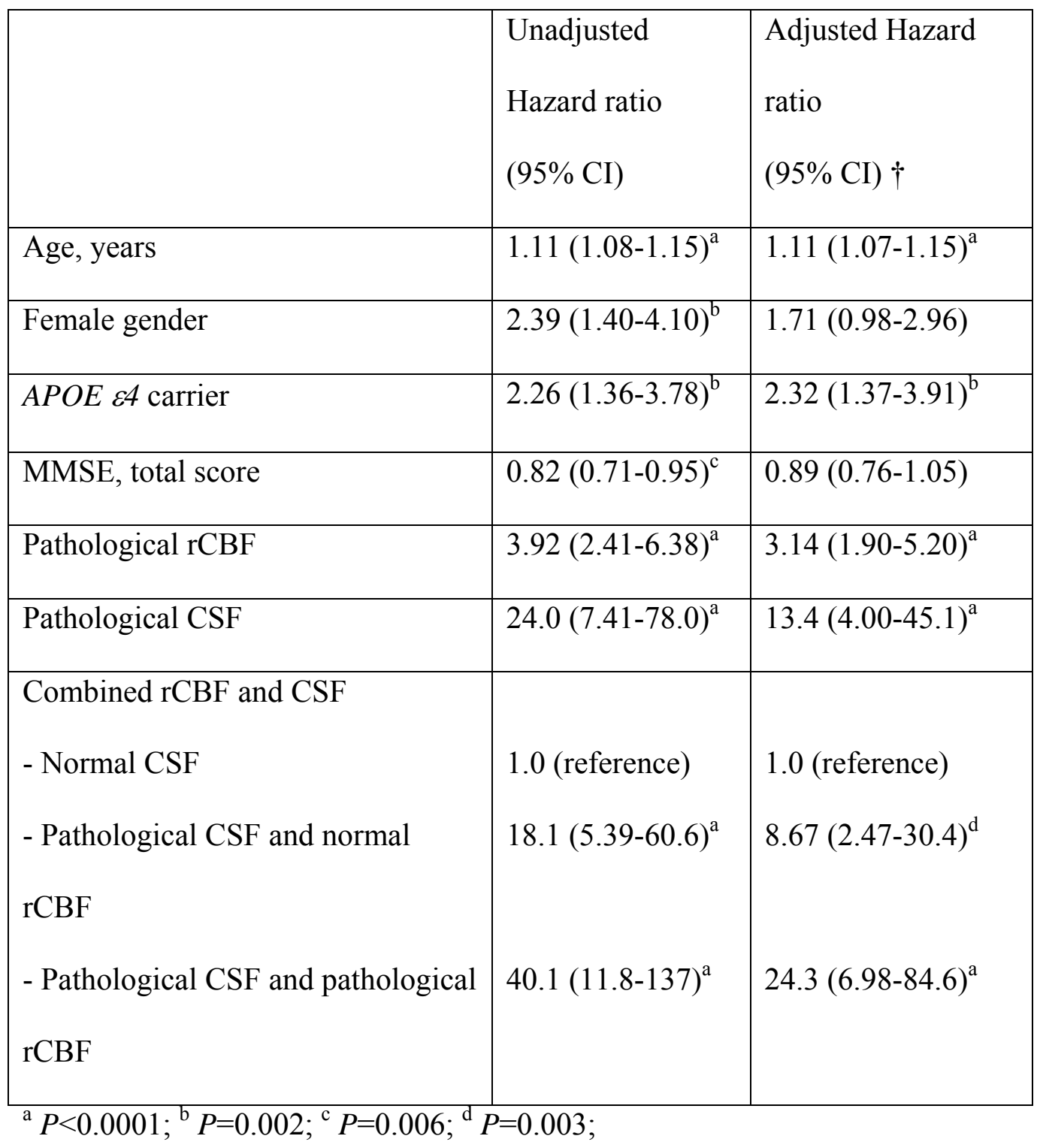

All data were collected at baseline. Abbreviations: APOE, apolipoprotein E; MMSE, MiniMental State Examination. Pathological rCBF was defined as a significantly decreased cortical $\mathrm{rCBF}$ in the parietal areas representing more than $1.5 \%$ of the total cerebral cortex; Pathological CSF, a combination of CSF total tau $>350 \mathrm{ng} / \mathrm{L}$ and CSF A $\beta 42<530 \mathrm{ng} / \mathrm{L}$. $\uparrow$ Adjusted (if applicable) for the baseline demographic variables, age, sex, MMSE and APOE $\varepsilon 4$ carrier status. 
MCI-AD versus Controls
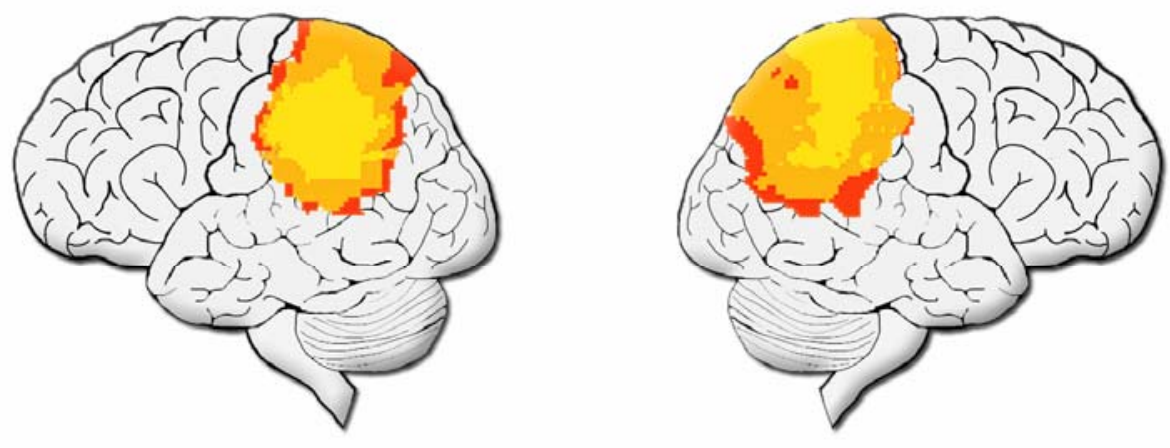

$$
\begin{gathered}
\begin{array}{c}
\text { Significance } \\
\text { level }
\end{array} \\
0.05- \\
0.01-
\end{gathered}
$$

MCI-AD versus Stable MCI
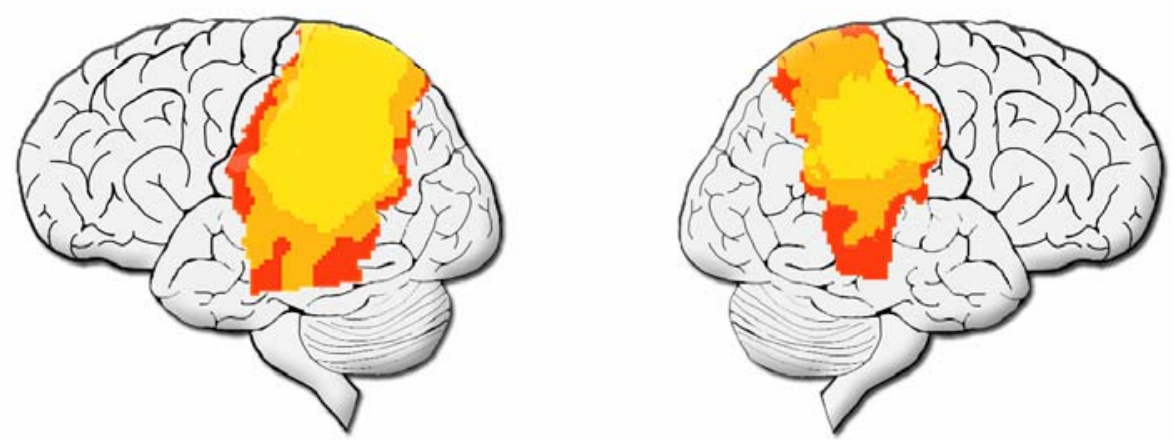

$0.001-$ 


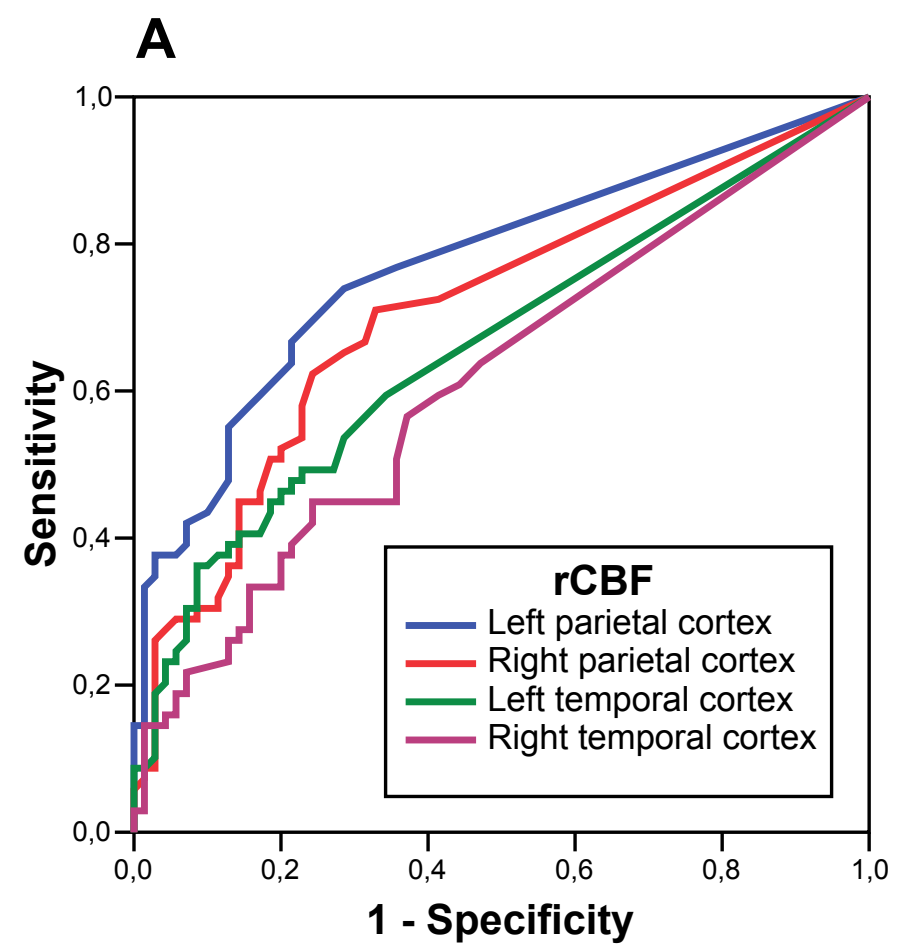

B

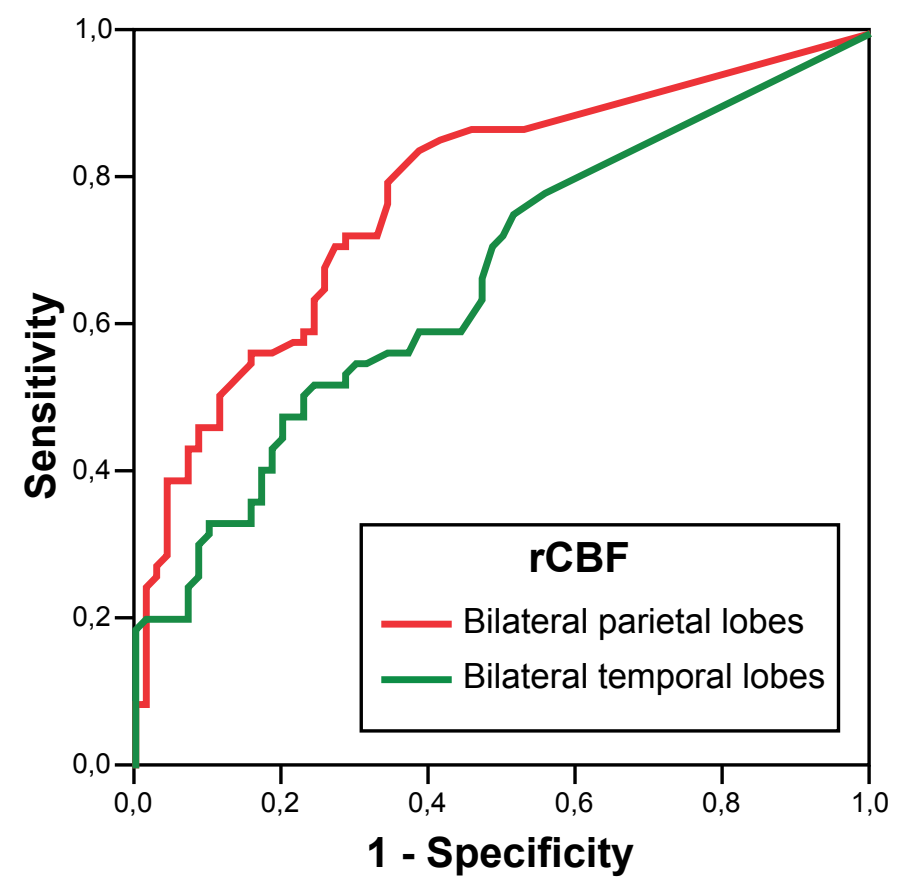

Figure 2 
A

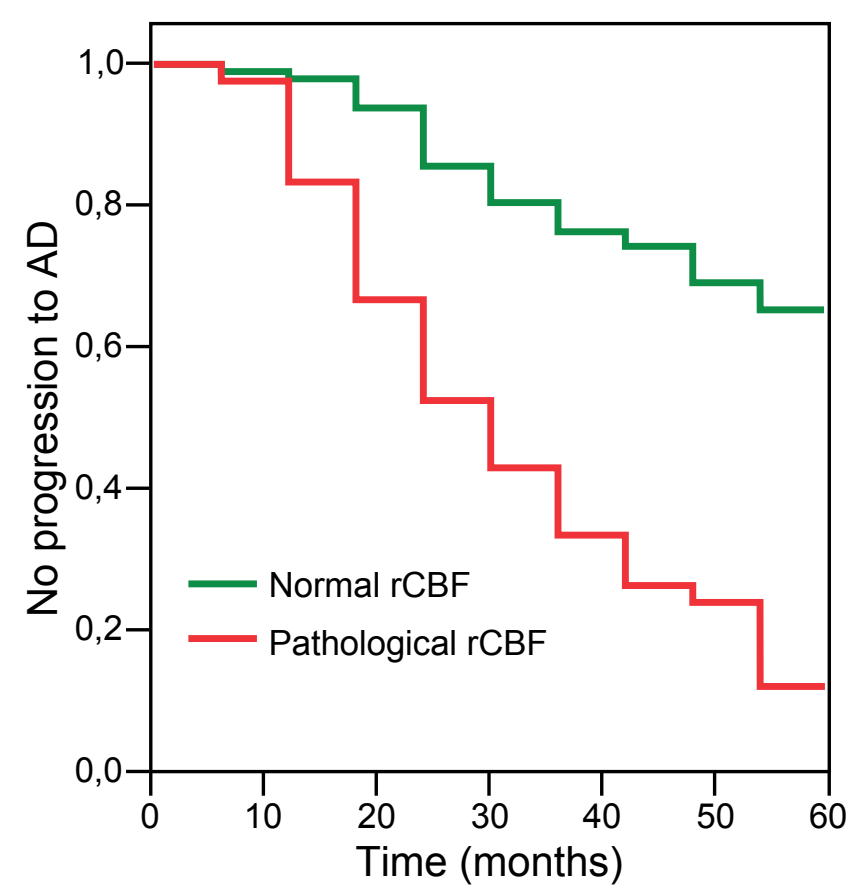

B

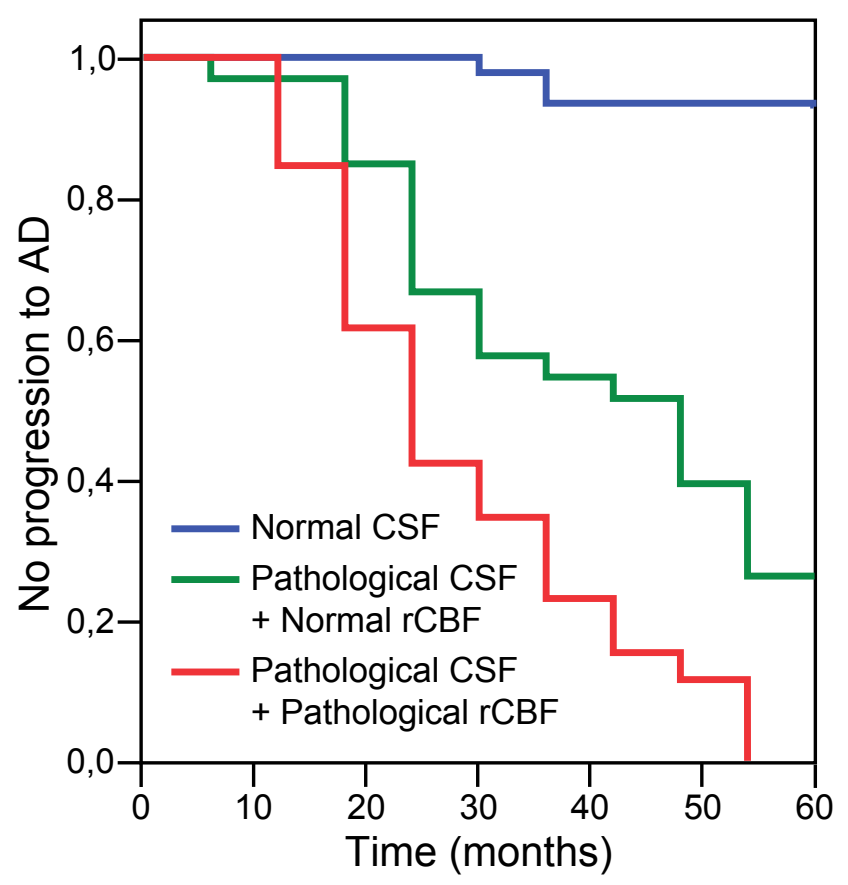

Figure 3 